\title{
In vivo SENSITIVITY OF Penicillium digitatum STRAINS INFECTING CITRUS FRUITS TO SIX FUNGICIDES AND THEIR COMBINATIONS
}

(Received: 26.3.2009)

\author{
By \\ Gh. J. M. Kanan \\ Department of Biological Sciences, Mu'tah University, Karak, Jordan
}

\begin{abstract}
The effects of six fungicides and their mixtures on in vivo growth of four Penicillium digitatum strains and the development of post-harvest green mould on artificially inoculated orange and lemon citrus fruits were evaluated. Regression analysis, one way ANOVA, and Post Hoc multiple comparisons were carried out to test the significance of these treatments on fungal growth. All fungicides (except Blin exa) completely inhibited the growth of strain dg6 (the most sensitive) invading orange fruits. Benomyl has completely inhibited the growth of strains dg6, dg5 and dg4 infecting lemon fruits. Ranvil has generated complete inhibition of strains dg6 and dg5 infecting lemon fruits. The Benomyl/Ranvil mixture showed synergistic effect against strains: $\operatorname{dg} 2$ (the least sensitive) infected lemon and orange fruits, dg4 and dg5, infected orange fruits, where complete inhibition to fungal growth was observed. A combined concentration of 100:500 $\mu \mathrm{g} \cdot \mathrm{mL}^{-1}$ of Topsin/Blin or Blin /Canvil mixture generated complete inhibition to the fungal growth as a result of synergistic effect against all strains infected both citrus fruit types.
\end{abstract}

Key words: chemical control, fungicide mixtures, fungicides, green mould, in vivo, lemon, orange, Penicillium digitatum.

\section{INTRODUCTION}

Citrus post-harvest green mould caused by Penicillium digitatum (Pers.) Sacc. is considered one of the most economically important universal diseases, which lead to spoilage of almost all kinds of mature citrus fruits (Plaza et al., 2004). The fungus invades the fruit more rapidly at room temperature and predominates in mixed infections causing approximately 60 to $80 \%$ of decay (Palou et al., 2001). Citrus industry relies heavily on the extensive use of chemical fungicides as standard practice for the control of post-harvest fungal decay of citrus fruits (Mc Grath, 2001 and Pramila and Dubey, 2004). Members of the Sterol demethylation inhibitor (DMI) group of fungicides such as Imazalil and $O$-phenyl phenol have remained the most routinely used ones in California citrus packing houses in particular (Holmes and Eckert, 1999) and worldwide (Savocchia et al., 2004 and Sugiura et al., 2006). Furthermore, the DMI members have greatly inhibited the growth of Ascomycota and basidiomycota members (Savocchia et al., 2004; Ma et al., 2006). The benzimidazole precursor fungicides (azoles) such as Benomyl and Topsin $\mathrm{M}$ are ultimate broad-spectrum systemic fungicides (Tsuda et al., 2004) which showed selective toxicity to several fungal diseases, and considered of the most effective against penicillium moulds (Zamin et al., 1999 and Valiuskaite et al., 2006). However, the widespread and recurrent use of chemicals having the same active gradients in commercial packing houses had lead to loss of their effectiveness, resulting in proliferation of resistant strains to these fungicides (Bus et al., 1991; McGrath, 2001and Surviliene and Dambrauskiene, 2006). The occurrence of serious resistance problems had enhanced searching for alternative decay control options (Palou et al., 2002 and Irtwange, 2006).

The current investigation aimed at participation in overcoming these threats through in vivo evaluation of four combined concentrations from each of seven fungicide mixtures. The hope in such strategy is to improve decay resistance at reduced risks and achieving cost saving benefits through reducing fruit spoilage and extending shelf life.

\section{MATERIALS AND METHODS}

2.1. Penicillium digitatum strains and medium Conidiospores of four $P$. digitatum strains (dg2, 
$\operatorname{dg} 4, \operatorname{dg} 5$, and dg6) were obtained from infected orange (Citrus sinensis Osbeck) and lemon (Citrus limon Burm .f.) fruits, collected from distributors in Irbid and Al-Karak cities in Jordan. Conidiospores from purified single colonies were used as a source of pure cultures. Routine Aspergillus growth medium and handling techniques were used as described by Cove (1966) with slight modifications, i.e. $\mathrm{pH}$ 5.5; supplemented with $10 \mathrm{mM}$ glutamic acid and 10 $\mathrm{gL}^{-1}$ fructose as $\mathrm{C}$ - source.

\subsection{Tested fungicides}

Six fungicides were tested these are: (i) Bayfidan Turf - 25\% EC (Vydan), containing 25\% $(\mathrm{w} / \mathrm{v})$ triadimenol $\left(\mathrm{C}_{14} \mathrm{H}_{18} \mathrm{CIN}_{3} \mathrm{O}_{3}\right)$ as an active gradient and produced by Vapco. Company Jordan Blin exa - 5\% Sc containing 5\% (w/v) hexaconazole $\left(\mathrm{C}_{14} \mathrm{H}_{17} \mathrm{CI}_{2} \mathrm{~N}_{3} \mathrm{O}\right)$ as an active gradient and produced by IQV - Spain. Canvil $5 \%$ contains $5 \%(\mathrm{w} / \mathrm{v})$ hexaconazole and produced by Vapco. Company - Jordan. Ranvil - 5\% contains also hexaconazole $5 \% \quad(\mathrm{w} / \mathrm{v})$ and produced by Chem.Vet - Jordan Benomyl - 50\% W.P contains Benlate $50 \%(\mathrm{w} / \mathrm{w})$ with the formula $\mathrm{C}_{14} \mathrm{H}_{18} \mathrm{~N}_{4} \mathrm{O}_{3}$ and produced by Vapco. Company Jordan. (vi) Topsin M - 70\% W.P contains thiophanate-methyl $70 \%(\mathrm{w} / \mathrm{w})$ with the formula $\mathrm{C}_{12} \mathrm{H}_{14} \mathrm{~N}_{4} \mathrm{O}_{4} \mathrm{~S}_{2}$ and produced by Nippon Soda Japan.

\section{3. Citrus fruit types}

Two citrus fruit species at ripening maturity were used in this study and these are orange (Citrus sinensis Osbeck) and lemon fruits (Citrus limon Burm .f.).

\subsection{Surface sterilization of fruits and inoculation with conidiospores and fungicides}

Fruits were washed under running tap water for $5 \mathrm{~min}$. This was followed by surface sterilization with $0.6 \%$ sodium hypochlorite solution for 10 min. After that fruits were washed three times (5 min / each) with sterilized water inside a UV sterilized culture room. Surface sterilized fruits were immediately inoculated in a laminar flow cabinet placed in a UV sterilized culture room. Each fruit was wounded two wounds [each of $5 \mathrm{x}$ $5 \mathrm{~mm}$. for orange fruits (thick coat) and approximately half that for lemon fruits without causing leakage of juice] at the equatorial side with sterilized stainless steel scalpel. $15 \mu \mathrm{L}$ of conidiospores suspension $\left(10^{8}\right.$ spore $\left.\mathrm{mL}^{-1}\right)$ from any of the tested strains was inoculated into each wound under aseptic conditions. Two hours later eight different concentrations $(10,25,50,100$, $200,300,500$, and $\left.1000 \mu \mathrm{g} . \mathrm{mL}^{-1}\right)$ from any of the tested fungicides were tested by inoculating $15 \mu \mathrm{L}$ from each concentration into one wound (Palou et al., 2001). Control fruits were inoculated with sterilized distilled water. Three replicates were used per each treatment for each fruit type and each test was repeated at least twice. The treated fruits were sealed in sterilized transparent nylon bags and incubated at room temperature (22-25 ${ }^{\circ} \mathrm{C}$; optimal range for fungal growth) for two weeks then assessed thereafter for decay or infection symptoms.

\subsection{Inoculation of fruits with conidiospores and fungicides mixture}

The above mentioned procedure was carried out using four combined concentrations (50:50, 100:100, 100:500, and 500:1000 $\mu \mathrm{g} . \mathrm{mL}^{-1}$ ) from each fungicide mixture against fungal strains, where these mixtures showed homogenous solution without precipitation. Seven fungicide mixtures were used these are: Benomyl/Ranvil; Topsin M/Vydan; Vydan/Canvil; Blin exa/Canvil; Topsin M/Blin exa; Blin exa/Vydan; Topsin M/Canvil. Each combined concentration of fungicides mixture was loaded to the same wound, where three replicates from each fruit type were used for each combination and each treatment was repeated at least twice then fruits decay was assessed as mentioned before.

\subsection{Estimation of fruit surface area}

Since the fruit has an ellipsoid shape, which has greater polar radius (a) than the equatorial (b) one $(a>b)$ then the quantity $(e)=\sqrt{ }\left(1-b^{2} / a^{2}\right)$ is the eccentricity of the ellipse (Anton, 1995) and the surface area $(\mathrm{S})$ of the prolate ellipsoid is given by the following equation:

$\left.\mathrm{S}=2 \pi \mathrm{b}^{2}[1+(\mathrm{a} / \mathrm{b}) \operatorname{arcsine}(\mathrm{e}) / \mathrm{e})\right]$

Arcsine $(\mathrm{e})=\tan ^{-1}\left\{\mathrm{e} / \sqrt{ } 1-\mathrm{e}^{2}\right\}$

so, the polar as well as the equatorial radius of each fruit were measured, then both measurements were applied to a mathematical equation which was introduced into a visual basic computer program, in order to calculate the fruit surface area, and the percentage of cleared surface area.

\subsection{Statistical analysis}

The $\mathrm{IC}_{50}$ values were calculated by regression analysis for the relationship between the size of inhibition zone $(\mathrm{mm}$.) and the fungicide concentration (Log value) using Microsoft Excel 2003 and the SPSS program version 10. One way ANOVA was carried out to determine the significant effect of each of the seven fungicide mixtures on sizes of inhibition zones of the studied strains. This was followed by Post Hoc mul330tiple comparisons to determine the significance level of combined concentrations of 
fungicide mixtures and their interactions on sizes of inhibition zones of $P$. digitatum strains.

\section{RESULTS}

\subsection{In vivo sensitivity of $P$. digitatum strains to} some fungicides

Results of regression analysis indicate that there was a significant correlation (at the 0.01 level-2 tailed) between fungicide concentration $\left(\mu \mathrm{g} . \mathrm{mL}^{-1}\right)$ and the size of inhibition zone $(\mathrm{mm}$.) for the tested strains invading lemon and orange fruits (Table 1). All fungicides completely inhibited the growth of strain $\operatorname{dg} 6$ invading orange fruits where, the obtained $\mathrm{IC}_{50}$ values have ranged from $35 \mu \mathrm{g}$. $\mathrm{mL}^{-1}$ (with Benomyl Topsin) to 230 $\mu \mathrm{g} . \mathrm{mL}^{-1}$ with Canvil (Table 1). However, Benomyl was the only fungicide which showed complete inhibition $\left(\mathrm{IC}_{50}=375 \mu \mathrm{g} \cdot \mathrm{mL}^{-1}\right)$ of growth to strain $\mathrm{dg} 4$ on orange fruits (Table 1). In addition, Benomyl fungicide completely inhibited the growth of strains dg6, dg5 and dg4 infecting lemon fruits (Fig. 1b) and the obtained $\mathrm{IC}_{50}$ values of the three strains were 30,237 and $132.5 \mu \mathrm{g}$. $\mathrm{mL}^{-1}$, respectively. Furthermore, Ranvil (a hexaconazole member) has also generated complete inhibition of strains $\operatorname{dg} 6$ and dg5 (Fig. $1 b$ ) invading lemon fruits and the obtained $\mathrm{IC}_{50}$ values were 138 and $144 \mu \mathrm{g}$. $\mathrm{mL}^{-1}$ respectively. On the other hand, none of the tested fungicides caused completely inhibit the growth of strain dg2 (The least sensitive strain) on lemon fruits where, the obtained zones of inhibition at the concentration of $1000 \mu \mathrm{g} . \mathrm{mL}^{-1}$ have ranged from $7.0 \pm 2.30 \mu \mathrm{g} . \mathrm{mL}^{-1}$ with Vydan (The least effective fungicide) to $42.5 \pm 3.57 \mu \mathrm{g}$. $\mathrm{mL}^{-1}$ with Benomyl (Table 1).

\subsection{In vivo sensitivity of $P$. digitatum strains to} various combined concentrations of fungicides mixtures

One way analysis of variance (ANOVA) indicated that the fungicide mixtures (except Benomyl/Ranvil and Topsin/Blin exa) have significantly $(\mathrm{P}=0.000)$ affected the sizes of inhibition zones of the four tested fungal strains infecting both lemon and orange fruits.

\subsection{Schaffe multiple comparisons}

3.3.1. Effect of benomyl/ranvil mixture on growth of $P$. digitatum strains

The Benomyl/Ranvil mixture showed synergistic effect at all tested concentrations against strain dg2 infecting both lemon and orange fruits, where complete inhibition $(100 \%$ cleared fruit surface area - CSA) of fungal growth was obtained (Table 2). The same mixture (Benomyl/Ranvil) showed also, synergistic effect against strains dg4 and dg5 infecting orange fruits (complete inhibition was achieved at a concentration of 100:500 $\mu \mathrm{g} . \mathrm{mL}^{-1}$ ). In contrast, the same mixture of fungicides resulted in antagonistic effects against strains dg4 and dg6 infecting lemon fruits where the obtained maximum zones of inhibition were 15 and 50 $\mathrm{mm}$., respectively, and this resulted in 5.83 and $88.97 \%$ (CSA), respectively (Table 2). In addition, obtained results indicate that there was a significant difference $(\mathrm{P}=0.000)$ between the combined concentration 50:50 $\mu \mathrm{g} . \mathrm{mL}^{-1}$ of Benomyl/Ranvil mixture and the rest of combinations (100:100;100:500 and 500:1000 $\mu \mathrm{g}$. $\mathrm{mL}^{-1}$ ) on size of inhibition zones of strains dg4 and dg6 infecting lemon, but not orange $(\mathrm{P}=1.000)$ fruits. Furthermore, there was significant difference $(\mathrm{P}=0.000)$ between the combined concentration of 100:100 $\mu \mathrm{g} . \mathrm{mL}^{-1}$ and each of the following combinations: 100:500 and 500:1000 $\mu \mathrm{g} . \mathrm{mL}^{-1}$ on zone size of strain $\mathrm{dg} 5$ which infects both lemon (Fig. 1b) and orange fruits.

\subsubsection{Effect of topsin $M / C a n v i l$ mixture}

Results indicate that Topsin/Canvil mixture has generated synergistic effects against strains dg4 and dg5 infecting both fruit types, whereas, antagonistic effect was obtained against strain dg2 infecting both fruit types (Table 2). However, additive effects were obtained against zones of strain dg6, whether invading orange or lemon fruits where, all treatments have generated complete inhibition of fungal growth on orange fruits but not on lemon. Furthermore, results indicate that there was a significant difference between combined concentrations of 50:50 and $100: 500 \mu \mathrm{g} . \mathrm{mL}^{-1}$ on zones of strain dg4 infecting both citrus fruit types $(\mathrm{P}=0.000$ for both fruit types). Also, there was a significant difference between the combination of 100:100 $\mu \mathrm{g} . \mathrm{mL}^{-1}$ and each of the following combinations: 100:500 $(\mathrm{P}=0.002$ for lemon; $\mathrm{P}=0.001$ for orange) and 500:1000 $\mu \mathrm{g} \mathrm{mL}^{-1}$ ( $\mathrm{P}=0.000$ for both fruit types) on zones of strain dg5 infecting lemon and orange fruits, and on zones of strain dg6 infecting lemon $(\mathrm{p}=0.000)$ but not orange $(\mathrm{p}=1.000)$ fruits. Moreover, there was a significant difference between the combined concentrations of 100:100 and 500:1000 $\mu \mathrm{g} . \mathrm{mL}^{-1}$ on zones of strain $\mathrm{dg} 2$ infecting lemon $(\mathrm{P}=0.000)$ and orange $(\mathrm{P}=0.039)$ fruits, respectively.

\subsubsection{Effect of Topsin/Vydan mixture}

There was a significant difference between all combinations of applied concentrations (50:50; $100: 100 ; 100: 500$ and $500: 1000 \mu \mathrm{g} . \mathrm{mL}^{-1}$ ) of 
Table (1): In vivo sensitivity of four Penicillium digitatum strains to six fungicides, 14 days after incubation at $22-25^{\circ} \mathrm{C}$.

\begin{tabular}{|c|c|c|c|c|c|c|}
\hline $\begin{array}{l}\text { Fungicide/ } \\
\text { Fruit type }\end{array}$ & $\begin{array}{l}\text { Fungicide/ } \\
\text { Conc/range } \\
\left(\mu \mathrm{g} \mathrm{mL}^{-1}\right) \dagger\end{array}$ & $\begin{array}{r}\text { Fungal } \\
\text { strain }\end{array}$ & $\begin{array}{r}\text { Mean inhibition zone } \\
\text { (range) } \\
(\mathbf{m m}) \pm \mathrm{SD}\end{array}$ & $\mathrm{IC}_{50}$ & $\begin{array}{r}\text { Coeff } \\
\text { (r-value) }\end{array}$ & $\begin{array}{r}\text { Sig } \\
\text { (2-tailed) }\end{array}$ \\
\hline Benomyl/ & $10-25$ & $\operatorname{dg} 6$ & $14 \pm 4.24-17 \pm 4.46$ & & $0.797 *$ & 0.018 \\
\hline Orange & $50-1000$ & & $\mathrm{Cit}$ & 35 & & \\
\hline Benomyl/ & $10-25$ & $\operatorname{dg} 6$ & $34.5 \pm 2.33-39.5 \pm 4.74$ & & $0.801 *$ & 0.017 \\
\hline Lemon & $50-1000$ & & $\mathrm{Ci}$ & 30 & & \\
\hline Topsin M/ & $10-25$ & $\operatorname{dg} 6$ & $15 \pm 1.41-18.5 \pm 2.12$ & & $0.892 * *$ & 0.003 \\
\hline Orange & $50-1000$ & & $\mathrm{Ci}$ & 35 & & \\
\hline $\begin{array}{r}\text { Topsin M/ } \\
\text { Lemon }\end{array}$ & $10-1000$ & $\operatorname{dg} 6$ & $0.0-22.5 \pm 2.11$ & & $0.906^{* *}$ & 0.002 \\
\hline Ranvil/ & $10-100$ & $\operatorname{dg} 6$ & $4 \pm 2.82-9 \pm 2.88$ & & $0.874 * *$ & 0.005 \\
\hline Orange & $200-1000$ & & $\mathrm{Ci}$ & 145 & & \\
\hline Ranvil/ & $10-100$ & $\operatorname{dg} 6$ & $5 \pm 7.07-18.5 \pm 2.12$ & & $0.892 * *$ & 0.003 \\
\hline Lemon & $200-1000$ & & $\mathrm{Ci}$ & 138 & & \\
\hline Vydan/ & $10-100$ & $\operatorname{dg} 6$ & $10 \pm 2.32-30 \pm 1.65$ & & $0.913 * *$ & 0.002 \\
\hline Orange & $200-1000$ & & $\mathrm{Ci}$ & 128 & & \\
\hline Vydan/ & $10-1000$ & $\operatorname{dg} 6$ & 0.0 & & & \\
\hline Lemon & & & & & & \\
\hline Canvil/ & $10-200$ & $\operatorname{dg} 6$ & $2.0 \pm 2.82-28 \pm 4.48$ & 230 & $0.886 * *$ & 0.003 \\
\hline Orange & $300-1000$ & & $\mathrm{Ci}$ & & & \\
\hline $\begin{array}{l}\text { Canvil/ } \\
\text { Lemon }\end{array}$ & $10-1000$ & $\operatorname{dg} 6$ & $0.0-15 \pm 3.12$ & & $0.902 * *$ & 0.002 \\
\hline $\begin{array}{r}\text { Blin exa/ } \\
\text { Orange }\end{array}$ & $10-1000$ & $\operatorname{dg} 6$ & $6.5 \pm 0.70-44.5 \pm 2.12$ & & $0.893 * *$ & 0.003 \\
\hline $\begin{array}{r}\text { Blin exa/ } \\
\text { Lemon }\end{array}$ & $10-1000$ & $\operatorname{dg} 6$ & $0.0-23 \pm 2.82$ & & $0.982 * *$ & 0.000 \\
\hline $\begin{array}{r}\text { Benomyl/ } \\
\text { Orange }\end{array}$ & $10-1000$ & dg5 & $11 \pm 1.65-21.5 \pm 2.21$ & & $0.982 * *$ & 0.000 \\
\hline Benomyl/ & $10-200$ & $\operatorname{dg} 5$ & $4 \pm 1.44-21.5 \pm 2.20$ & 237 & $0.862 * *$ & 0.006 \\
\hline Lemon & $300-1000$ & & $\mathrm{Ci}$ & & & \\
\hline $\begin{array}{r}\text { Topsin M/ } \\
\text { Orange }\end{array}$ & $10-1000$ & $\operatorname{dg} 5$ & $0.0-13.5 \pm 4.99$ & & $0.905 * *$ & 0.002 \\
\hline $\begin{array}{r}\text { Topsin M/ } \\
\text { Lemon }\end{array}$ & $10-1000$ & $\operatorname{dg} 5$ & $0.0-15 \pm 8.48$ & & $0.955^{* *}$ & 0.000 \\
\hline $\begin{array}{l}\text { Ranvil/ } \\
\text { Orange }\end{array}$ & $10-1000$ & $\operatorname{dg} 5$ & $4.5 \pm 0.77-13.5 \pm 3.56$ & & $0.980 * *$ & 0.000 \\
\hline Ranvil/ & $10-100$ & $\operatorname{dg} 5$ & $4 \pm 1.42-11 \pm 1.43$ & 144 & $0.879 * *$ & 0.004 \\
\hline Lemon & $200-1000$ & & $\mathrm{Ci}$ & & & \\
\hline $\begin{array}{l}\text { Vydan/ } \\
\text { Orange }\end{array}$ & $10-1000$ & dg5 & $4.0 \pm 2.23-13.0 \pm 3.32$ & & $0.875^{* *}$ & 0.004 \\
\hline $\begin{array}{l}\text { Vydan/ } \\
\text { Lemon }\end{array}$ & $10-1000$ & $\operatorname{dg} 5$ & $0.0-0.0$ & & & \\
\hline $\begin{array}{l}\text { Canvil/ } \\
\text { Orange }\end{array}$ & $10-1000$ & $\operatorname{dg} 5$ & $1.0 \pm 1.41-8.5 \pm 0.77$ & & $0.959 * *$ & 0.000 \\
\hline $\begin{array}{l}\text { Canvil/ } \\
\text { Lemon }\end{array}$ & $10-1000$ & $\operatorname{dg} 5$ & $3.0 \pm 1.11-8.5 \pm 2.87$ & & $0.980 * *$ & 0.000 \\
\hline $\begin{array}{r}\text { Blin exa/ } \\
\text { Orange }\end{array}$ & $10-1000$ & $\operatorname{dg} 5$ & $5.0 \pm 1.41-16.5 \pm 2.13$ & & $0.952 * *$ & 0.000 \\
\hline $\begin{array}{r}\text { Blin exa/ } \\
\text { Lemon }\end{array}$ & $10-1000$ & dg5 & $1.5 \pm 2.12-11.0 \pm 3.11$ & & $0.991 * *$ & 0.000 \\
\hline $\begin{array}{r}\text { Benomyl/ } \\
\text { Orange }\end{array}$ & $10-1000$ & $\operatorname{dg} 2$ & $5.0 \pm 1.43-37.0 \pm 1.71$ & & $0.953 * *$ & 0.000 \\
\hline $\begin{array}{r}\text { Benomyl/ } \\
\text { Lemon }\end{array}$ & $10-1000$ & $\operatorname{dg} 2$ & $15.0 \pm 1.44-42.5 \pm 3.57$ & & $0.986 * *$ & 0.000 \\
\hline $\begin{array}{r}\text { Topsin M/ } \\
\text { Orange }\end{array}$ & $10-1000$ & $\operatorname{dg} 2$ & $0.0 \pm 15.0 \pm 2.83$ & & $0.984 * *$ & 0.000 \\
\hline
\end{tabular}

( Continued) 
Table (1): Continued.

\begin{tabular}{|c|c|c|c|c|c|c|}
\hline $\begin{array}{l}\text { Fungicide/ } \\
\text { Fruit type }\end{array}$ & $\begin{array}{r}\text { Fungicide/ } \\
\text { Conc/range } \\
\left(\mu \mathrm{g} \mathrm{mL}^{-1}\right) \dagger\end{array}$ & $\begin{array}{r}\begin{array}{c}\text { Fungal } \\
\text { strain }\end{array}\end{array}$ & $\begin{array}{r}\text { Mean inhibition zone } \\
\text { (range) } \\
(\mathrm{mm}) \pm \mathrm{SD}\end{array}$ & $\mathrm{IC}_{50}$ & $\begin{array}{c}\text { Coeff } \\
\text { (r-value) }\end{array}$ & $\begin{array}{r}\text { Sig } \\
\text { (2-tailed) }\end{array}$ \\
\hline $\begin{array}{r}\text { Topsin M/ } \\
\text { Lemon }\end{array}$ & $10-1000$ & $\operatorname{dg} 2$ & $3.5 \pm 4.95-35 \pm 1.88$ & & $0.972 * *$ & 0.000 \\
\hline $\begin{array}{l}\text { Ranvil/ } \\
\text { Orange }\end{array}$ & $10-1000$ & dg2 & $4.5 \pm 0.71-20.5 \pm 3.55$ & & $0.972 * *$ & 0.000 \\
\hline $\begin{array}{l}\text { Ranvil/ } \\
\text { Lemon }\end{array}$ & $10-1000$ & $\operatorname{dg} 2$ & $3.5 \pm 2.21-15.5 \pm 3.53$ & & $0.981 * *$ & 0.000 \\
\hline $\begin{array}{l}\text { Vydan/ } \\
\text { Orange }\end{array}$ & $10-1000$ & $\operatorname{dg} 2$ & $0.0-6.0 \pm 1.36$ & & $0.791 *$ & 0.019 \\
\hline $\begin{array}{l}\text { Vydan/ } \\
\text { Lemon }\end{array}$ & $10-1000$ & dg2 & $0.0-7.0 \pm 2.30$ & & 0.553 & 0.155 \\
\hline $\begin{array}{l}\text { Canvil/ } \\
\text { Orange }\end{array}$ & $10-1000$ & dg2 & $4.0 \pm 1.61-36.0 \pm 2.36$ & & $0.944 * *$ & 0.000 \\
\hline $\begin{array}{l}\text { Canvil/ } \\
\text { Lemon }\end{array}$ & $10-1000$ & $\operatorname{dg} 2$ & $14.0 \pm 2.61-40.0 \pm 3.34$ & & $0.986 * *$ & 0.000 \\
\hline $\begin{array}{r}\text { Blin exa/ } \\
\text { Orange }\end{array}$ & $10-1000$ & $\operatorname{dg} 2$ & $3.0 \pm 1.41-16.0 \pm 5.65$ & & $0.958 * *$ & 0.000 \\
\hline $\begin{array}{l}\text { Blin exa/ } \\
\text { Lemon }\end{array}$ & $\begin{array}{r}10-100 \\
200-1000\end{array}$ & dg2 & $\begin{array}{r}4.0 \pm 1.44-9.5 \pm 1.77 \\
\mathrm{Ci}\end{array}$ & 143 & $0.875 * *$ & 0.004 \\
\hline $\begin{array}{r}\text { Benomyl/ } \\
\text { Orange }\end{array}$ & $\begin{array}{r}10-300 \\
500-1000\end{array}$ & $\operatorname{dg} 4$ & $8.5 \pm 0.77-19.5 \pm 2.02$ & 375 & $0.765^{*}$ & 0.027 \\
\hline $\begin{array}{r}\text { Benomyl/ } \\
\text { Lemon }\end{array}$ & $\begin{array}{r}10-100 \\
200-1000\end{array}$ & $\operatorname{dg} 4$ & $15.5 \pm 0.71-25.5 \pm 2.12$ & 132.5 & $0.886 * *$ & 0.003 \\
\hline $\begin{array}{r}\text { Topsin } \mathrm{M} / \\
\text { Orange }\end{array}$ & $10-1000$ & $\operatorname{dg} 4$ & $0.0-14.5 \pm 4.95$ & & $0.908 * *$ & 0.002 \\
\hline $\begin{array}{r}\text { Topsin M/ } \\
\text { Lemon }\end{array}$ & $10-1000$ & $\operatorname{dg} 4$ & $0.0-19.0 \pm 1.44$ & & $0.959 * *$ & 0.000 \\
\hline $\begin{array}{l}\text { Ranvil/ } \\
\text { Orange }\end{array}$ & $10-1000$ & $\operatorname{dg} 4$ & $4.0 \pm 1.41-9.5 \pm 2.61$ & & $0.962 * *$ & 0.000 \\
\hline $\begin{array}{l}\text { Ranvil/ } \\
\text { Lemon }\end{array}$ & $10-1000$ & $\operatorname{dg} 4$ & $0.0-14 \pm 4.24$ & & $0.969 * *$ & 0.000 \\
\hline $\begin{array}{l}\text { Vydan/ } \\
\text { Orange }\end{array}$ & $10-1000$ & $\operatorname{dg} 4$ & $0.0-15 \pm 1.42$ & & $0.950 * *$ & 0.000 \\
\hline $\begin{array}{l}\text { Vydan/ } \\
\text { Lemon }\end{array}$ & $10-1000$ & $\operatorname{dg} 4$ & $0.0-20.5 \pm 2.70$ & & $0.987 * *$ & 0.000 \\
\hline $\begin{array}{l}\text { Canvil/ } \\
\text { Orange }\end{array}$ & $10-1000$ & dg4 & $4.5 \pm 1.76-14.0 \pm 2.83$ & & $0.964 * *$ & 0.000 \\
\hline $\begin{array}{l}\text { Canvil/ } \\
\text { Lemon }\end{array}$ & $10-1000$ & $\operatorname{dg} 4$ & $0.0-19.5 \pm 2.12$ & & $0.959 * *$ & 0.000 \\
\hline $\begin{array}{r}\text { Blin exa/ } \\
\text { Orange }\end{array}$ & $10-1000$ & $\operatorname{dg} 4$ & $4.0 \pm 1.41-27.5 \pm 3.53$ & & $0.820 *$ & 0.013 \\
\hline $\begin{array}{r}\text { Blin exa/ } \\
\text { Lemon }\end{array}$ & $10-1000$ & $\operatorname{dg} 4$ & $0.0-57.0 \pm 6.81$ & & $0.827 *$ & 0.011 \\
\hline
\end{tabular}


Topsin/Vydan mixture ( $\mathrm{P}$ values were within the range of $\mathrm{P}=0.000$ to $\mathrm{P}=0.039$ ) on size of inhibition zones of the four strains (except strain dg5 infecting lemon fruits; $P$ values were within the range of $\mathrm{P}=0.193$ to $\mathrm{P}=0.693$ ) infecting both lemon (Fig. 1a) and orange fruits. Synergistic effects against zones of strain dg2 infecting orange fruits (inhibition zone $=32 \mathrm{~mm}$. equivalent to $19 \%$ CSA) and strain dg4 infecting both orange (inhibition zone $=53 \mathrm{~mm}$. equivalent to $56 \%$ CSA) and lemon (56 $\mathrm{mm}$; equivalent to $85 \%$ CSA) fruits were obtained. However, additive effects against zones of strain dg2 infecting lemon fruits (Maximum zones of $46 \mathrm{~mm}$; equivalent to $84 \%$ CSA) were obtained. In contrast, antagonistic effects were seen against zones of strain dg6 infecting orange fruits, (maximum zone was $30 \mathrm{~mm}$ as compared to complete inhibition with the singly tested fungicides (Table 1).

\subsubsection{Effect of Vydan/Canvil mixture}

Table (2). Indicates that no significant difference between the combination of 100:500 $\mu \mathrm{g} . \mathrm{mL}^{-1}$ and 500:1000 $\mu \mathrm{g}$. $\mathrm{mL}^{-1}$ on inhibition zone of strains dg4; dg5 and dg6 infecting orange fruits (Fig. $2 \mathrm{~b}$ and $\mathrm{d}$ ). The obtained P-values were $1.000 ; 0.693$; and 1.0 , respectively. Furthermore, synergistic effects were obtained against zone of strains dg4 infecting orange fruits (complete inhibition was obtained at a combined concentration of 100:500 $\mu \mathrm{g} . \mathrm{mL}^{-1}$ ) and strain dg5 infecting both fruit types (47.4\% CSA on lemon as compared to inhibition zones in the range of 0.0 $-8.5 \mathrm{~mm}$. with the singly used fungicides). Also, synergistic effects were obtained against zones of strain dg6 infecting lemon $(46.48 \%$ CSA as compared to $0.0 \mathrm{~mm}$. inhibition zone with Vydan fruits). However, additive effects against zones of strain dg6 infecting orange fruits were obtained, where complete inhibition was achieved with the combined concentration of 100:500 $\mu \mathrm{g} . \mathrm{mL}^{-1}$ (Table 2).

\subsubsection{Effect of Topsin/Blin exa mixture}

Topsin/Blin mixture recorded synergistic effect against zones of strains $\mathrm{dg} 2, \mathrm{dg} 4, \mathrm{dg} 5$ and dg6, (Table 2) where complete inhibition at a combined concentration of 100:500 $\mu \mathrm{g}$. $\mathrm{mL}^{-1}$ was obtained against strains $\mathrm{dg} 2$ (infecting orange and lemon fruits), dg4 (infecting lemon) and $\operatorname{dg} 6$ (infecting both fruit types). However, such mixture has caused complete inhibition to strain dg4 (infecting orange), at all tested concentrations ( Table 2 ). There was no significant difference Between the combination of 100:500 and 500:
$1000 \mu \mathrm{g} . \mathrm{mL}^{-1}$ against strains $\mathrm{dg} 2 ; \mathrm{dg} 4$ and $\mathrm{dg} 6$ infecting both fruit types (Fig. 2a).

\subsubsection{Effect of blin exa/vydan mixture}

This mixture of fungicides resulted in synergistic effect against zones of strains $\mathrm{dg} 2$, dg4; and dg6 infecting both orange and lemon fruits, where, complete inhibition of fungal growth was generated with the combination of 100:500 $\mu \mathrm{g} . \mathrm{mL}^{-1}$ on orange and lemon fruits, except strain $\mathrm{dg} 2$, where an inhibition zone of $16 \mathrm{~mm}$. (9\% CSA) was obtained (Table 2). However, there were significant differences among the combined concentrations ( $\mathrm{P}$ values within the range of $\mathrm{P}=0.000$ to $\mathrm{P}=0.039$ ) on zones of the four strains, infecting both fruit types (Fig. 2c) with exception of the combinations of 100:500 $\mu \mathrm{g} . \mathrm{mL}^{-1}$ and 500:1000 ( $\mathrm{P}$ values have ranged from 0.693 to 1.000).

\subsubsection{Effect of Blin exa/Canvil mixture}

Results indicate that synergistic effects were obtained against zones of the four strains, where the growth of strains $\operatorname{dg} 2$ and $\operatorname{dg} 6$ on both fruit types was completely inhibited at the combined concentration of 100:500 $\mu \mathrm{g}$. $\mathrm{mL}^{-1}$ (Table 2). However, the combination of 500:1000 $\mu \mathrm{g} . \mathrm{mL}^{-1}$ resulted in complete inhibition to strain $\mathrm{dg} 4$ infecting both fruit types (Table 2), and strain dg5 infecting orange fruits, whereas, the growth of the same strain on lemon fruits was completely inhibited at a concentration of 100:500 $\mu \mathrm{g} . \mathrm{mL}^{-1}$ (Table 2). Moreover, results indicate that there was no significant difference between combined concentration of 100:500 $\mu \mathrm{g} . \mathrm{mL}^{-1}$ and 500:1000 $\mu \mathrm{g} . \mathrm{mL}^{-1}$ on zones of strains: $\operatorname{dg} 2 \quad(\mathrm{P}=1.000)$ infecting both fruit types; dg5 infecting lemon $(\mathrm{P}=1.000)$ and $\operatorname{dg} 6$.

\subsubsection{Correlation between fungicides mixture and size of inhibition zones of $P$. digitatum strains}

Results of regression analysis indicate significant correlation (at the 0.01 level: 2-tailed) between applied combined concentrations of: Topsin/Vydan; Vydan/Canvil; Blin exa/Vydan; and Blin exa/Canvil mixtures of fungicides and inhibition zones of the four tested strains, whether such strains grown on lemon or orange fruits. Furthermore, the applied combined concentrations of Benomyl/Ranvil mixture showed significant correlation (at the 0.01 level: 2-tailed) with zones of strains: $\operatorname{dg} 4$ infecting lemon fruits; $\operatorname{dg} 5$ infecting both citrus fruit types; and dg6 infecting lemon fruits. 
Table (2) :Percentage of cleared (spore-free) citrus fruit (orange and lemon) surface area as an effect of different combined concentrations of fungicides mixtures.

\begin{tabular}{|c|c|c|c|c|c|c|c|c|c|c|}
\hline \multirow{2}{*}{$\begin{array}{l}\text { Fungicides } \\
\text { mixture }\end{array}$} & \multirow{2}{*}{$\begin{array}{l}\text { Combined. } \\
\text { conc } \\
\left(\mu \mathrm{g} \mathrm{mL}^{-1}\right)^{\mathrm{a}}\end{array}$} & \multicolumn{4}{|c|}{ orange } & \multicolumn{5}{|c|}{ Lemon } \\
\hline & & $\begin{array}{l}\mathrm{IZ}^{\mathrm{b}} \\
(\mathrm{cm})\end{array}$ & $\begin{array}{l}\mathrm{IZA}^{\mathrm{c}} \\
\left(\mathrm{cm}^{2}\right)\end{array}$ & $\begin{array}{l}\mathrm{SA}^{\mathrm{d}} \\
\left(\mathrm{cm}^{2}\right)\end{array}$ & $\begin{array}{l}\mathrm{CSA}^{\mathrm{e}} \\
(\%)\end{array}$ & $\begin{array}{l}\mathrm{IZ} \\
(\mathrm{cm})\end{array}$ & $\begin{array}{l}\text { IZA } \\
\left(\mathrm{cm}^{2}\right)\end{array}$ & $\begin{array}{l}\mathrm{SA} \\
\left(\mathrm{cm}^{2}\right)\end{array}$ & $\begin{array}{l}\text { CSA } \\
(\%)\end{array}$ & $\begin{array}{l}\text { Fungal } \\
\text { strain }\end{array}$ \\
\hline \multirow{12}{*}{$\begin{array}{l}\text { Benomyl/ } \\
\text { Ranvil }\end{array}$} & $1 ; 2 ; 3 ; 4$ & $\mathrm{Ci}^{\mathrm{f}}$ & & 216.4 & 100 & $\mathrm{Ci}$ & & 119.43 & 100 & dg2 \\
\hline & 1 & $\mathrm{Ci}$ & & \multirow[t]{4}{*}{178.29} & 100 & 1 & 3.14 & \multirow[t]{4}{*}{121.23} & 2.6 & \multirow[t]{4}{*}{$\operatorname{dg} 4$} \\
\hline & 2 & $\mathrm{Ci}$ & & & 100 & 1.4 & 6.15 & & 5.08 & \\
\hline & 3 & $\mathrm{Ci}$ & & & 100 & 1.4 & 6.15 & & 5.08 & \\
\hline & 4 & $\mathrm{Ci}$ & & & 100 & 1.5 & 7.07 & & 5.83 & \\
\hline & 1 & 0.5 & 0.79 & \multirow[t]{3}{*}{187.86} & 0.42 & 1.4 & 6.15 & \multirow[t]{3}{*}{89.2} & 6.9 & \multirow[t]{3}{*}{$\operatorname{dg} 5$} \\
\hline & 2 & 0.9 & 2.54 & & 1.35 & 2.5 & 19.63 & & 22.00 & \\
\hline & $3 ; 4$ & $\mathrm{Ci}$ & & & 100 & $\mathrm{Ci}$ & & & 100 & \\
\hline & 1 & $\mathrm{Ci}$ & & \multirow[t]{4}{*}{184.62} & 100 & 2.4 & 18.09 & \multirow[t]{4}{*}{88.23} & 20.50 & \multirow[t]{4}{*}{$\operatorname{dg} 6$} \\
\hline & 2 & $\mathrm{Ci}$ & & & 100 & 2.5 & 19.63 & & 22.24 & \\
\hline & 3 & $\mathrm{Ci}$ & & & 100 & 3 & 28.26 & & 32.03 & \\
\hline & 4 & $\mathrm{Ci}$ & & & 100 & 5 & 78.50 & & 88.97 & \\
\hline Topsin/ & 1 & 1.1 & 0.95 & 161.32 & 0.59 & 1.5 & 7.07 & 84.71 & 8.34 & $\operatorname{dg} 2$ \\
\hline \multirow[t]{15}{*}{ Canvil } & 2 & 1.5 & 1.77 & & 1.10 & 2 & 12.56 & & 14.83 & \\
\hline & 3 & 1.5 & 1.77 & & 1.10 & 2 & 12.56 & & 14.83 & \\
\hline & 4 & 1.8 & 2.55 & & 1.58 & 3 & 28.26 & & 33.36 & \\
\hline & 1 & 1.8 & 2.54 & 55.59 & 4.58 & 0.9 & 2.54 & 53.47 & 4.76 & $\mathrm{dg} 4$ \\
\hline & 2 & 2.1 & 3.46 & & 6.23 & 1.7 & 9.08 & & 16.97 & \\
\hline & 3 & 2.7 & 5.73 & & 10.29 & 2.3 & 16.61 & & 31.07 & \\
\hline & 4 & 3.6 & 10.18 & & 18.30 & 3.1 & 30.18 & & 56.43 & \\
\hline & 1 & 0.5 & 0.20 & 53.59 & 0.37 & 1.5 & 7.07 & 25.22 & 28.01 & $\operatorname{dg} 5$ \\
\hline & 2 & 1.3 & 1.33 & & 2.48 & 1.8 & 10.17 & & 40.34 & \\
\hline & 3 & 1.9 & 2.83 & & 5.29 & 2.3 & 16.61 & & 65.86 & \\
\hline & 4 & 2.3 & 4.15 & & 7.75 & 2.6 & 21.23 & & 84.17 & \\
\hline & 1 & $\mathrm{Ci}$ & & 204.42 & 100 & 1.3 & 5.31 & 100.84 & 5.26 & $\operatorname{dg} 6$ \\
\hline & 2 & $\mathrm{Ci}$ & & & 100 & 1.6 & 8.04 & & 7.97 & \\
\hline & 3 & $\mathrm{Ci}$ & & & 100 & 2.2 & 15.20 & & 15.07 & \\
\hline & 4 & $\mathrm{Ci}$ & & & 100 & 2.5 & 19.63 & & 19.46 & \\
\hline Blin/ & 1 & 0.9 & 0.64 & 207.8 & 0.31 & 0.8 & 2.01 & 88.12 & 2.28 & $\operatorname{dg} 2$ \\
\hline Vydan & 2 & 1.3 & 1.33 & & 0.64 & 1.2 & 4.52 & & 5.12 & \\
\hline & 3 & $\mathrm{Ci}$ & & & 100 & 1.5 & 7.07 & & 8.01 & \\
\hline & 4 & $\mathrm{Ci}$ & & & 100 & 1.6 & 8.04 & & 9.11 & \\
\hline & 1 & 1.8 & 2.54 & 42.23 & 6.03 & 2.8 & 24.62 & 63.01 & 39.07 & $\operatorname{dg} 4$ \\
\hline & 2 & 2.4 & 4.52 & & 10.71 & 3.3 & 34.19 & & 54.27 & \\
\hline & $3 ; 4$ & $\mathrm{Ci}$ & & & 100 & $\mathrm{Ci}$ & & & 100 & \\
\hline & 1 & 1.8 & 2.54 & 62.67 & 4.06 & 0.4 & 0.503 & 25.22 & 1.99 & $\operatorname{dg} 5$ \\
\hline & 2 & 2.1 & 3.46 & & 5.53 & 1.1 & 3.80 & & 15.07 & \\
\hline & $3 ; 4$ & $\mathrm{Ci}$ & & & 100 & $\mathrm{Ci}$ & & & 100 & \\
\hline & 1 & 0.6 & 0.28 & 158.35 & 0.18 & 1.0 & 3.14 & 98.39 & 3.19 & $\operatorname{dg} 6$ \\
\hline & 2 & 0.8 & 0.50 & & 0.32 & 1.1 & 3.80 & & 3.86 & \\
\hline & 3 & 1.0 & 0.79 & & 0.50 & 1.4 & 6.15 & & 6.26 & \\
\hline & 4 & 1.1 & 0.95 & & 0.60 & 1.5 & 7.07 & & 7.18 & \\
\hline Topsin/ & 1 & 1.3 & 1.33 & 39.20 & 3.38 & 2.8 & 24.62 & 77.39 & 31.81 & $\operatorname{dg} 2$ \\
\hline Blin & 2 & 1.9 & 2.83 & & 7.23 & 3.7 & 42.99 & & 55.55 & \\
\hline & $3 ; 4$ & $\mathrm{Ci}$ & & & 100 & $\mathrm{Ci}$ & & & 100 & \\
\hline & 1 & $\mathrm{Ci}$ & & 204.44 & 100 & 1.8 & 10.18 & 156.57 & 6.50 & $\operatorname{dg} 4$ \\
\hline & 2 & $\mathrm{Ci}$ & & & 100 & 2.0 & 12.56 & & 8.02 & \\
\hline & $3 ; 4$ & $\mathrm{Ci}$ & & & 100 & $\mathrm{Ci}$ & & & 100 & \\
\hline & 1 & 1.5 & 1.77 & 99.20 & 1.78 & 1.9 & 11.34 & 88.26 & 12.84 & $\operatorname{dg} 5$ \\
\hline & 2 & 2.1 & 3.46 & & 3.49 & 2.6 & 21.23 & & 24.05 & \\
\hline & 3 & 3.3 & 8.55 & & 8.62 & 3.6 & 40.69 & & 46.11 & \\
\hline & 4 & 5.2 & 21.23 & & 21.40 & 4.7 & 69.36 & & 78.59 & \\
\hline & 1 & 3.2 & 8.04 & 42.58 & 18.89 & 1.3 & 5.31 & 22.33 & 23.76 & $\operatorname{dg} 6$ \\
\hline & 2 & 4.1 & 13.20 & & 30.99 & 2.4 & 18.09 & & 81.0 & \\
\hline & $3 ; 4$ & $\mathrm{Ci}$ & & & 100 & $\mathrm{Ci}$ & & & 100 & \\
\hline
\end{tabular}

( Continued) 


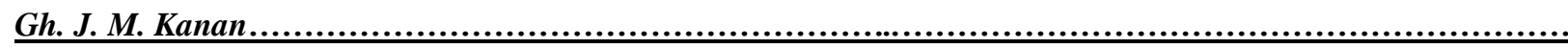

Table (2): Continued.

\begin{tabular}{|c|c|c|c|c|c|c|c|c|c|c|}
\hline \multirow{2}{*}{$\begin{array}{l}\text { Fungicides } \\
\text { mixture }\end{array}$} & \multirow{2}{*}{$\begin{array}{l}\text { Combined. } \\
\text { conc } \\
\left(\mu \mathrm{g} \mathrm{mL}^{-1}\right)^{\mathrm{a}}\end{array}$} & \multicolumn{4}{|c|}{ orange } & \multicolumn{5}{|c|}{ Lemon } \\
\hline & & $\begin{array}{l}\mathrm{IZ}^{\mathrm{b}} \\
(\mathrm{cm})\end{array}$ & $\begin{array}{l}\mathrm{IZA}^{\mathrm{c}} \\
\left(\mathrm{cm}^{2}\right)\end{array}$ & $\begin{array}{l}\mathrm{SA}^{\mathrm{d}} \\
\left(\mathrm{cm}^{2}\right)\end{array}$ & $\begin{array}{l}\mathrm{CSA}^{\mathrm{e}} \\
(\%)\end{array}$ & $\begin{array}{l}\mathrm{IZ} \\
(\mathrm{cm})\end{array}$ & $\begin{array}{l}\mathrm{IZA} \\
\left(\mathrm{cm}^{2}\right)\end{array}$ & $\begin{array}{l}\mathrm{SA} \\
\left(\mathrm{cm}^{2}\right)\end{array}$ & $\begin{array}{l}\text { CSA } \\
(\%)\end{array}$ & $\begin{array}{l}\text { Fungal } \\
\text { strain }\end{array}$ \\
\hline Vydan/ & 1 & 1.1 & 0.95 & 36.67 & 2.59 & 0.7 & 1.54 & 24.04 & 6.40 & dg2 \\
\hline \multirow[t]{4}{*}{ Canvil } & 2 & 1.9 & 2.84 & & 7.73 & 1.3 & 5.31 & & 22.07 & \\
\hline & 3 & 2.1 & 3.46 & & 9.45 & 1.8 & 10.17 & & 42.32 & \\
\hline & 4 & 2.6 & 5.31 & & 14.47 & 2.1 & 13.85 & & 57.60 & \\
\hline & 1 & 0.3 & 0.071 & 191.12 & 0.037 & 0.7 & 1.54 & 68.38 & 2.25 & $\mathrm{dg} 4$ \\
\hline Vydan/ & 2 & 0.4 & 0.13 & & 0.066 & 0.8 & 2.01 & & 2.94 & \\
\hline \multirow[t]{10}{*}{ Canvil } & 3 & $\mathrm{Ci}$ & & & 100 & 1.0 & 3.14 & & 4.59 & \\
\hline & 4 & $\mathrm{Ci}$ & & & 100 & 1.3 & 5.31 & & 7.76 & \\
\hline & 1 & 1.4 & 1.54 & 55.59 & 2.77 & 0.7 & 1.54 & 55.75 & 2.76 & dg5 \\
\hline & 2 & 1.8 & 2.54 & & 4.58 & 1.2 & 4.52 & & 8.11 & \\
\hline & 3 & 3.3 & 8.55 & & 15.38 & 2.3 & 16.61 & & 29.79 & \\
\hline & 4 & 3.4 & 9.07 & & 16.32 & 2.9 & 26.41 & & 47.37 & \\
\hline & 1 & 2.3 & 4.15 & 51.08 & 8.13 & 0.3 & 0.28 & 21.89 & 1.29 & $\operatorname{dg} 6$ \\
\hline & 2 & 3.4 & 9.07 & & 17.77 & 0.9 & 2.54 & & 11.62 & \\
\hline & 3 & $\mathrm{Ci}$ & & & 100 & 1.3 & 5.31 & & 24.24 & \\
\hline & 4 & $\mathrm{Ci}$ & & & 100 & 1.8 & 10.17 & & 46.48 & \\
\hline Topsin/ & 1 & 0.3 & 0.071 & 41.45 & 0.17 & 1.2 & 4.52 & 79.57 & 5.68 & dg2 \\
\hline \multirow[t]{15}{*}{ Vydan } & 2 & 0.8 & 0.50 & & 1.21 & 1.9 & 11.34 & & 14.25 & \\
\hline & 3 & 1.7 & 2.27 & & 5.47 & 3.7 & 42.99 & & 54.02 & \\
\hline & 4 & 3.2 & 8.04 & & 19.39 & 4.6 & 66.44 & & 83.50 & \\
\hline & 1 & 0.7 & 0.38 & 39.19 & 0.98 & 1.4 & 6.15 & 119.57 & 5.15 & $\mathrm{dg} 4$ \\
\hline & 2 & 1.9 & 2.83 & & 7.23 & 3.1 & 30.18 & & 25.24 & \\
\hline & 3 & 3.2 & 8.04 & & 20.51 & 4.2 & 55.39 & & 46.32 & \\
\hline & 4 & 5.3 & 22.05 & & 56.27 & 5.6 & 100 & & 85.32 & \\
\hline & 1 & 0.9 & 0.64 & 48.58 & 1.31 & 1.6 & 8.04 & 58.07 & 13.84 & dg5 \\
\hline & 2 & 1.2 & 1.13 & & 2.33 & 1.7 & 9.07 & & 15.63 & \\
\hline & 3 & 1.8 & 2.54 & & 5.24 & 1.7 & 9.07 & & 15.63 & \\
\hline & 4 & 2.1 & 3.46 & & 7.13 & 1.8 & 10.17 & & 17.52 & \\
\hline & 1 & 1.5 & 1.77 & 178.27 & 0.99 & 1.1 & 3.8 & 58.08 & 6.54 & $\operatorname{dg} 6$ \\
\hline & 2 & 1.8 & 2.54 & & 1.43 & 1.6 & 8.04 & & 13.84 & \\
\hline & 3 & 2.0 & 3.14 & & 1.76 & 2.1 & 13.85 & & 23.84 & \\
\hline & 4 & 3.0 & 7.07 & & 3.96 & 3.2 & 32.15 & & 55.36 & \\
\hline Blin/ & 1 & 2.3 & 4.15 & 48.53 & 8.56 & 2.4 & 18.09 & 57.39 & 31.51 & $\mathrm{dg} 2$ \\
\hline \multirow[t]{14}{*}{ Canvil } & 2 & 2.9 & 6.60 & & 13.60 & 3.7 & 42.99 & & 74.90 & \\
\hline & $3 ; 4$ & $\mathrm{Ci}$ & & & 100 & $\mathrm{Ci}$ & & & 100 & \\
\hline & 1 & 1.5 & 1.77 & 39.19 & 4.51 & 2.1 & 13.85 & 93.52 & 14.81 & $\operatorname{dg} 4$ \\
\hline & 2 & 1.9 & 2.83 & & 7.23 & 3.2 & 32.15 & & 34.38 & \\
\hline & 3 & 3.8 & 11.34 & & 28.92 & 5.3 & 88.20 & & 94.31 & \\
\hline & 4 & $\mathrm{Ci}$ & & & 100 & $\mathrm{Ci}$ & & & 100 & \\
\hline & 1 & 0.9 & 0.64 & 88.23 & 0.72 & 1.0 & 3.14 & 39.66 & 7.92 & dg5 \\
\hline & 2 & 1.3 & 1.33 & & 1.50 & 1.1 & 3.8 & & 9.58 & \\
\hline & 3 & 2.8 & 6.15 & & 6.98 & $\mathrm{Ci}$ & & & 100 & \\
\hline & 4 & $\mathrm{Ci}$ & & & 100 & $\mathrm{Ci}$ & & & 100 & \\
\hline & 1 & 0.4 & 0.13 & 184.62 & 0.07 & 0.6 & 1.13 & 57.64 & 1.96 & dg6 \\
\hline & 2 & 0.5 & 0.20 & & 0.11 & 1.4 & 6.15 & & 10.68 & \\
\hline & 3 & $\mathrm{Ci}$ & & & 100 & 3.3 & 34.19 & & 59.32 & \\
\hline & 4 & $\mathrm{Ci}$ & & & 100 & $\mathrm{Ci}$ & & & 100 & \\
\hline
\end{tabular}




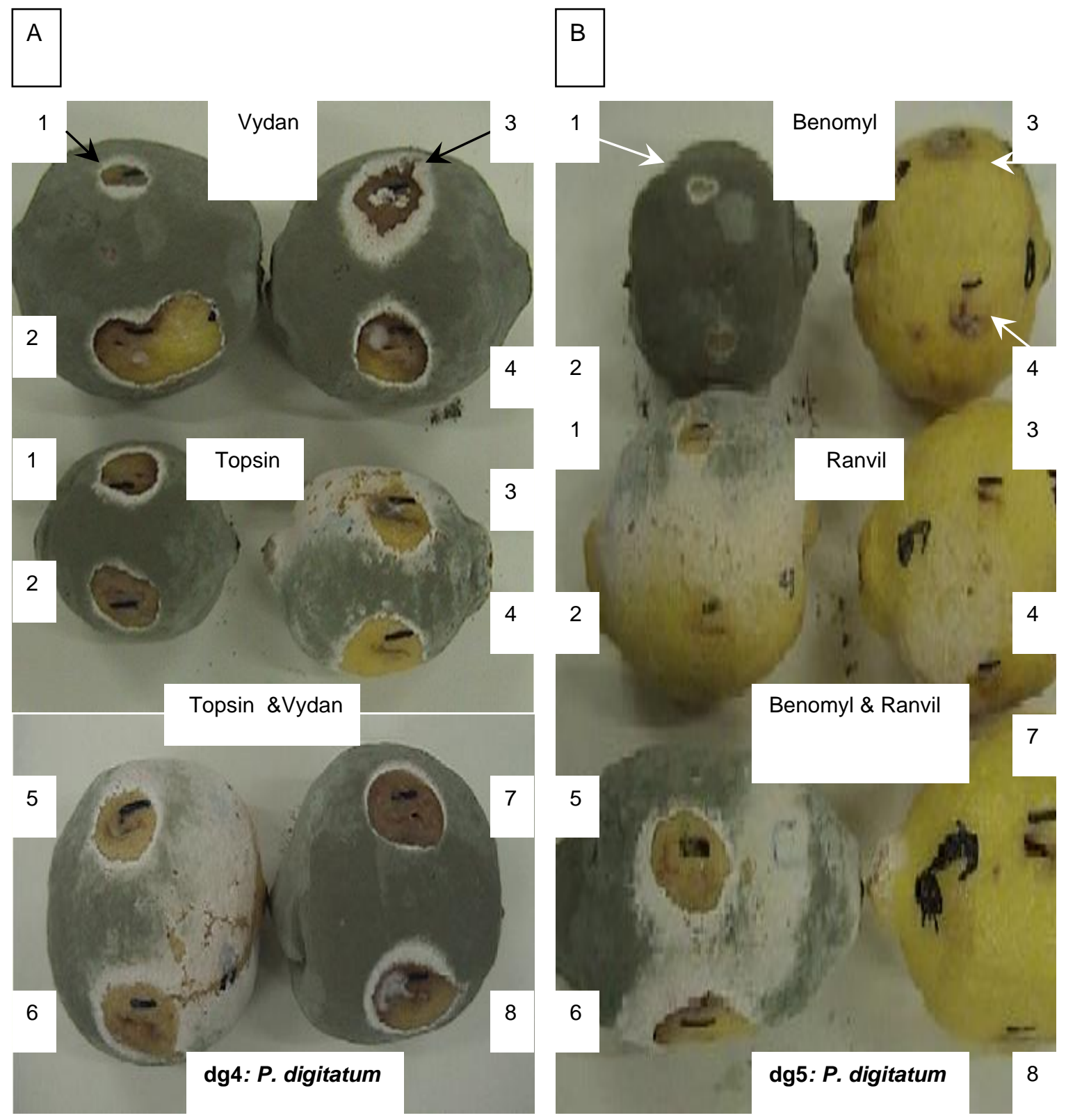

Fig. (1): In vivo sensitivity of two $P$. digitatum strains infecting lemon fruits to two fungicides and their combinations. Panel a: Inhibition zones generated by strain dg4 as the effect of treatment by Vydan; Topsin and a combination of both. Panel b: Effect of Benomyl; Ranvil; and a combination of both on growth of strain dg5 of $P$. digitatum.1: denotes for $50 \mu \mathrm{g} \mathrm{mL}^{-1} ; 2: 100 \mu \mathrm{g} \mathrm{mL}^{-1} ; 3: 500 \mu \mathrm{g} \mathrm{mL}^{-1} ; 4: 1000 \mu \mathrm{g}$ $\mathrm{mL}^{-1} ; 5 ; 6 ; 7 ; 8$ : denote for combined concentrations of 50:50; 100:100; 100:500; and 500:1000 $\mu \mathrm{g} \mathrm{mL}^{-1}$ from both fungicides respectively. Note complete inhibition to strain dg5 growth was obtained at concentrations of 500 and $1000 \mu \mathrm{g} \mathrm{mL}{ }^{-1}$ of Benomyl; and Ranvil. Also, a mixture of both generated complete inhibition for the same strain at combined concentrations of 100:500 and 500:1000 $\mu \mathrm{g} \mathrm{mL}^{-1}$. 


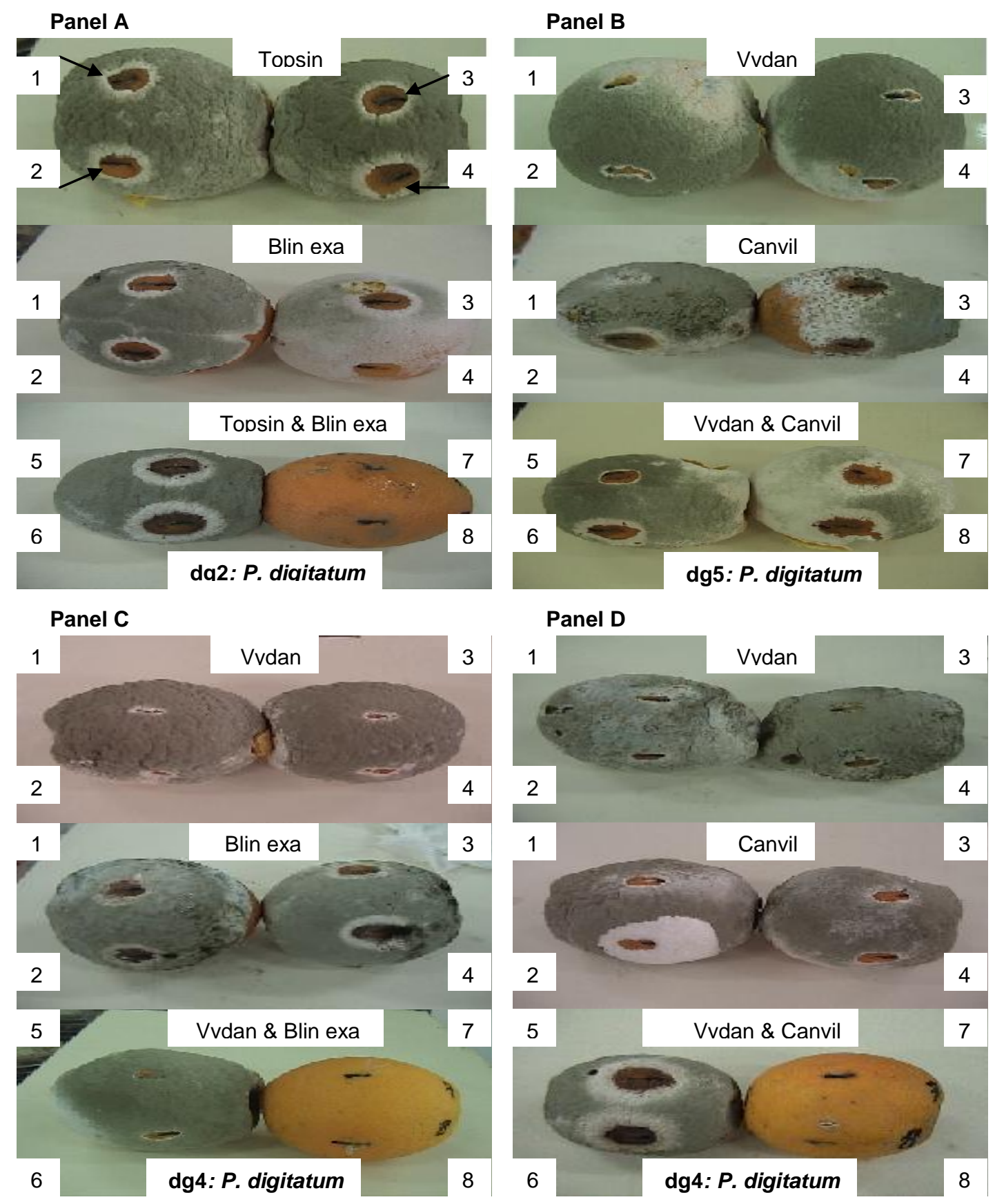

Fig. (2): In vivo sensitivity of three $P$. digitatum strains infecting orange fruits to four fungicides and their combinations. Panel a: Inhibition zones generated by strain dg2 as an effect of treatment by Topsin; Blin exa and a combination of both. Panel b: Effect of Vydan; Canvil; and a combination of both on growth of strain dg5. Panel c: Effect of Vydan; Blin exa and a combination of both on growth of strain dg4. Panel d: Effect of Vydan; Canvil and a combination of both on growth of strain dg4. Number 1: denotes for $50 \mu \mathrm{g} \mathrm{mL}^{-1}$; 2: $100 \mu \mathrm{g} \mathrm{mL}^{-1} ; 3: 500 \mu \mathrm{g} \mathrm{mL}^{-1}$; 4: $1000 \mu \mathrm{g} \mathrm{mL}^{-1}$; 5; 6; 7; 8: denote for combined concentrations of 50:50; 100:100; 100:500; and 500:1000 $\mu \mathrm{g} \mathrm{mL}$. ${ }^{1}$ from both fungicides respectively. Complete inhibition of strains $\mathrm{dg} 2$ and $\mathrm{dg} 4$ growth was obtained at combined concentrations of 100:500 and 500:1000 $\mu \mathrm{g} \mathrm{mL}^{-1}$ of Topsin \& Blin; Vydan \& Blin; and Vydan \& Canvil.

\section{DISCUSSION}


The recurrent multiplicity of the same active ingredient will lead to a major commercial problem of fungal resistance to such fungicides, which reflected serious difficulties in disease control (Cunningham, 2005 and Surviliene and Dambrauskiene, 2006). In this work, Ranvil (a DMI member) showed high efficacy in controlling the infection by $P$. digitatum strains (gives complete inhibition or the largest zones after Benomyl) than Canvil; Blin exa and Vydan although, all have the same active component (hexaconazole) but produced by different national and international companies under different trade marks. Furthermore, Vydan has shown to be the least effective, especially when strains infect lemon rather than orange fruits. These findings agreed with the results of in vitro study (Kanan, 2008) which revealed that Ranvil and Benomyl were the most effective against tested strains, whereas, Canvil was the least. However, these results disagreed with the findings of Lam and Lim (1993) and with that of Savocchia and his coworkers (2004) who stated that Vydan has shown an excellent control of white rust on Chrysanthemum and powdery mildew on roses and grapevines. The triazole fungicides are sterol demethylation inhibitors (DMI) that inhibit the enzyme, C14-demethylase, leading to depletion of ergosterol which serves as a bioregulators for membrane fluidity and integrity in fungal cells resulting in alteration in their cell walls (Ma et al., 2006 and Sugiura et al., 2006). Possible mechanisms leading to DMI resistance include mutations in the DMI target enzyme C14-alphademethylase (CYP51) which lead to decrease affinity of DMI to target protein (De'lye et al., 1997). Resistance to demethylation inhibitors (DMI) in biotypes of Penicillium species is thought to be controlled by a polygenic system (Van Tuyl, 1977 and Kalamarakis et al., 1987) where the development of resistance would appear due to cumulative or additive effects of mutations in several minor genes (De Waard et al., 1982 and Georgopoulos and Skylakakis, 1986). In this kind of resistance (i.e. quantitative or continuous resistance) pathogens exhibit a range of sensitivity to the fungicide, depending on the type and number of altered genes where, variations in sensitivity within the population is continuous, and selection occurs in a directional manner. Resistance in this case is seen as erosion of disease control that can be regained by either applying higher concentrations of the fungicide, or by more frequent use of the fungicide. However, the strain could be revert back to be sensitive if the fungicide is no longer used. Other mechanisms include over-expression of ATP binding cassette (ABC) transporters encoding efflux pumps that effectively pump toxic chemicals out of the cell and here the strain may gain resistance to several fungicides using the same mechanism (Hayashi et al., 2002 and Zwiers et al., 2002). Furthermore, the deposition of fungicide in lipid droplets and change in $\mathrm{pH}$ leading to protonation of fungicide is possible mechanism of resistance (McGrath, 2001). Obtained results showed that the benzimidazole systemic fungicide Benomyl (Benlate) was the most effective (mostly generates complete inhibition or the largest zones) in terms of restraining fungal growth. In contrast, the fungicide Topsin (TBZ member as benomyl) did not show complete hyphal growth inhibition, at a range of concentrations from $10^{-1}$ to $1000 \mu \mathrm{g}$. $\mathrm{mL}^{-1}$, with the four tested strains infecting both fruit types (except strain dg6 infecting orange fruits). These findings disagreed with the in vitro findings of Kanan (2008) also with that of Zamin and his co-workers (1999) who indicated that the systemic fungicide Topsin $\mathrm{M}$ has controlled several fungal diseases including powdery mildew, downy mildew which infect grapes and wheat leaf brown rust. Concerning Benomyl mode of action it is firstly transformed into methyl-2-benzimidazole carbamate metabolites, (carbendazim) that causes morphological distortion of germinating spores (Tsuda et al., 2004). This fungicide binds to microtubules inhibiting $\beta$-tubulin assembly and interferes with cell division (Dalgie, 2005). Furthermore, Benomyl shows selective toxicity to several microorganisms including fungi and interferes with intracellular transportation causing loss of membrane transport ability (Amar and Reinhold, 1973). Resistance to benzimidazole fungicides is thought to be a kind of qualitative type since it is resulted from modification of a single major gene, where pathogens became either resistant or sensitive to the fungicide and here disruptive selection occurs (Mc Grath, 2001). Resistance in this case is seen as complete loss of disease control, which resulted in conformational changes at the target site that can not be regained by using higher concentrations or more frequent fungicides application. This type of mutations resulted in high selection pressure during fungicides application and there is low selection pressure to remove them in absence of fungicide (Van Tuyl, 1977and Mc Grath, 2001). Concerning the effect of fungicides mixture, when Benomyl and Topsin 
$M$ were mixed with another fungicide from the DMI group, more controllable effects were obtained. These findings agreed with the in vitro study (Kanan, 2008) which indicated that inhibition zones of tested strains were significantly affected by fungicides mixture and complete inhibition was mostly obtained, especially when either Benomyl or Topsin M was mixed with a DMI member. These results agreed also with the suggestions of Shaw (1993) who stated that studies of resistance development revealed that the combination of two selective fungicides to combat resistance is reasonable strategy, only when used against wild population of the pathogen with an extremely low frequency of resistance to both fungicides. In addition, the sequential use of two unrelated fungicides may be more effective strategy, because, the application of fungicides mixture showing the same mode of action would lead to resistance as a result of positive cross resistance. However, when resistance to one chemical class lead to increase sensitivity to the other, this result is negative cross resistance. The mechanisms that underlie the development of fungicide resistance, have mainly proved to be some kind of modification of the biochemical target site in the pathogen, which render the site more sensitive to damage by combined fungicides. This suggestion agreed with the findings of Mc Grath (2001) also with that of Survilienė and Dambrauskienè (2006).

\section{Acknowledgments}

This work was supported by Mu'tah University (process number S. R/120/14/180). Special Thanks to Dr. Z. Alawneh and Mr. A. Al-Omari, Mutah University-Jordan for their help in designing computer program to calculate the fruit surface area.

\section{REFERENCES}

Amar L. and Reinhold L. (1973). Loss of membrane transportability in leaf cell and release of protein as a result of osmotic shock. Plant Physiol., 51: 620-625.

Anton H. (1995). Calculus with analytic geometry. John Wiley and Sons Inc, Newyork.

Bus V.G., Bongers A.J. and Risse L.A. (1991). Occurrence of Penicillium digitatum and $P$. italicum resistant to benomyl, thiabendazole and imazalil on citrus fruit from different geographic origins. Plant Dis., 75: 1098-1100.

Cove D.J. (1966). The induction and repression of nitrate reductase in the fungus Aspergillus nidulans. Biochem. Biophys Acta., 113: 5156.

Cunningham N. (2005). Registered fungicides for citrus post harvest-What is out there. Packer Newsletter., 77: 1-6.

Dalgie O.D.F. (2005). The effect of fungicide benomyl (benlate) on growth and mitosis in onion (Allium cepa L.) root apical meristem. Acta. Biol. Hung., 56(1-2): 119-128.

De'lye C., Laigret F. and Corio-Costet M. F. (1997). A mutation in the $14 \alpha$-demethylase gene of Uncinula necator that correlates with resistance to a sterol biosynthesis inhibitor. Appl. Environ. Microbiol., 63: 2966-2970.

De Waard M.A., Groeneweg H. and Van Nistelrooy J.G.M. (1982). Laboratory resistance to fungicides which inhibit ergosterol biosynthesis in Penicillium italicum. Neth. J. Plant Pathol., 88: 99-112.

Georgopoulos S.G. and Skylakakis G. (1986). Genetic variability in the fungi and problem of fungicide resistance. Crop Prot., 5: 299-305.

Hayashi K., Schoonbeek H. and De Waard M.A. (2002). Expression of the ABC transporter BcatrD from Botrytis cinerea reduces sensitivity to sterol demethylation inhibitor fungicides. Pestic. Biochem. Physiol., 73: 110-121.

Holmes G.J. and Eckert J. W. (1999). Sensitivity of Penicillium digitatum and P. Italicum to postharvest citrus fungicides in California. Phytopathol., 89: 716-721.

Irtwange S. V. (2006). Hot water treatment: a nonchemical alternative in keeping quality during post harvest handling of Citrus fruits. Agricultural engineering international. Invited Overview. CTGRE. J., 8(5): 1-10.

Kalamarakis A.E., Ziogas B.N. and Georgopoulos S.G. (1987). Resistance to ergosterol biosythesis inhibitor in Nectia haematococca var. cucurbitae. Chem. Control. Newsl., 8: 1819.

Kanan G.J.M. (2008). In vitro evaluation of Penicillium digitatum Sacc. Strains sensitivity to various fungicides from Jordan. Pakistan. J. Biol. Sci.,11(12): 1517-1529.

Lam C.H. and Lim T.K. (1993). Efficacy of hexaconazole for the control of white rust on chrysanthemum and powdery mildew on roses. Int. J. pest manag., 39 (2): 156-160.

Ma Z., Proffer T.J., Jacobs J.L. and Sundin G.W. (2006). Overexpression of the $14 \alpha$-Demethlase target gene (CYP51) mediates fungicide resistance in Blumeriella jaapii. Appl. Environ. Microbiol., 72: 2581- 2585. 
Mc Grath M.T. (2001). Fungicide resistance in Cucurbit Powdery Mildew: experiences and challenges. Plant Dis., 85: 236-245.

Palou L., Usall J., Smilanick J.L. and Vinas I. (2001). Control of postharvest blue and green molds of oranges by hot water, sodium carbonate, and sodium bicarbonate. Plant Dis., 85: 371-376.

Palou L., Usall J., Smilanick J.L., Aguilar M.J. and Vinas I. (2002). Evaluation of additives and low-toxicity compounds as alternative chemicals for the control of Penicillium digitatum and Penicillium italicum on citrus fruit. Pest. Manage. Sci., 58: 459-466.

Plaza P., Sanbruno A., Usall J., Lamarca N.,Torres R., Pons J. and Vinas I. (2004). Integration of curing treatments with degreening to control the main postharvest diseases of Calementine mandarins. Postharv. Biol. Technol., 34(1): 29-37.

Pramila T. and Dubey N. K. (2004). Exploitation of natural products as an alternative strategy to control postharvest fungal rotting of fruit and vegetables. Postharv. Biol. Technol., 32: 235-245.

Savocchia S., Stummer B.E., Wicks T.J.,Van Heeswijck R. and Scott E.S. (2004). Reduced sensitivity of Uncinula necator to sterol demethylation inhibiting fungicides in southern Australian vineyards. Aust. Plant Pathol., 33(4): 465-473.

Shaw M.W. (1993). Theoretical analysis of the effect of interacting activities on the rate of selection for combined resistance to fungicide mixtures. Crop Prot., 12: 120-125.
Sugiura H., Hayashi K., Tanaka T., Takenaka M. and Uesugi Y. (2006). Mutual antagonism between sterol demethylation inhibitors and phosphorothiolate fungicides on Pyricularia oryzae the implications for their mode of action. Pest. Sci., 39: 193-198.

Surviliene E. and Dambrauskiene E. (2006). Effect of different active ingredients of fungicides on Alternaria spp. Growth In vitro. Agro. Res. 4(special issue): 403-406.

Tsuda M., Itoh H. and Kato S. (2004). Evaluation of the systemic activity of simeconazole in comparison with that of other DMI fungicides. Pest Manag. Sci., 60(9): 875-880.

Valiuskaite A., Surviliene E., Lugauskas A. and Levinskaite L. (2006). Ecological aspects of distribution of potential toxin-producing micromycetes on stored apple fruit. Ekologija., 3: 60-63.

Van Tuyl J.M. (1977). Genetic aspects of resistance to imazalil in Aspergillus nidulans. Neth. J. Plant Pathol., 83(1): 169-176.

Zamin S.S., Soaliha A. and Shaukat S.S. (1999). Effect of systemic fungicide (Topsin-M) and inscticide (Dimecron) on germination, seedling growth and phenolic content of Pennisetum americanum L. Pakistan J. Biol. Sci., 2(1): 182-184.

Zwiers L., Stergiopoulos I., Van Nistelrooy J.G.M. and De Waard M.A. (2002). ABC transporters and azoles susceptibility in laboratory strains of the wheat pathogen Mycosphaerella graminicola. Antimicrob. Agents Chemother., 46: 3900-3906.
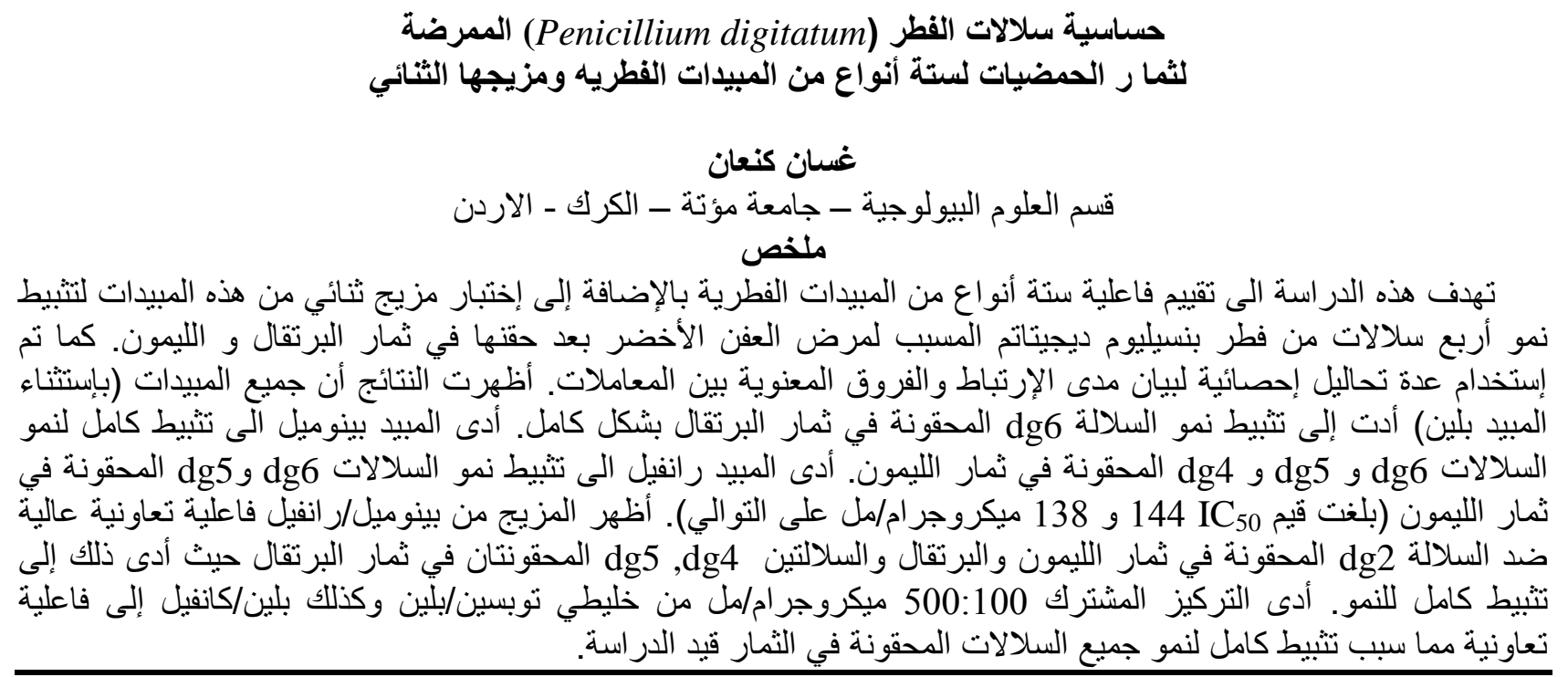

المجلة العلمية لكلية الزر اعة - جامعة القاهرة - المجلد (60) العدد الثالث (يوليو 2009 ) 329-341. 